\title{
Continuing the Saga of Fluffy Mirror Fermions: Lattice Regulating Chiral Gauge Theories
}

\author{
Dorota M. Grabowska*t \\ Berkeley Center for Theoretical Physics, University of California, Berkeley, CA 94720 \\ Theoretical Physics Group, Lawrence Berkeley National Laboratory, Berkeley, CA 94720 \\ E-mail: dgrabowska@berkeley . edu
}

\section{David B. Kaplan}

Institute for Nuclear Theory, Box 351550, Seattle, Washington 98195-1550, USA

E-mail: dbkaplan@uw.edu

\begin{abstract}
I discuss continuing work on a recent proposal for a nonperturbative regulator for chiral gauge theories that combines domain wall fermions and gradient flow. Implementing chiral gauge theories on the lattice requires not only decoupling mirror fermions to allow for fermions in complex representations, but also a road to failure for theories with fermions in anomalous representations. Unlike attempts to gap the mirror fermion spectrum, this proposal gives the mirror fermions exponentially soft form factors, allowing them to decouple from ordinary matter except through nontrivial gauge field topology. I discuss progress on various open questions for this formulation that were left unresolved in the original work.
\end{abstract}

34th annual International Symposium on Lattice Field Theory

24-30 July 2016

University of Southampton, $U K$

\footnotetext{
* Speaker.

${ }^{\dagger}$ This work was supported in by DOE Grant No. DE-FG02-00ER41132, an NSF Graduate Research Fellowship, Grant No. DGE-1256082 and NSF Grant No. 82759-13067-44-PHHXM
} 


\section{Introduction}

Nonperturbatively regulating chiral gauge theories has been a long-standing problem in quantum field theory. It may be that finding such a regulator reveals no new or unexpected behavior; however as there is currently no other method for gaining access to the nonperturbative regime, theoretical control of the full path integral is necessary for determining the full matter content of chiral gauge theories. Experimental probes of the Standard Model only provide tests of the weak coupled regime, via the Electroweak sector, and so only reveal the ingredients necessary for defining these theories perturbatively. Furthermore, while certain models for Beyond the Standard Model physics utilize the dynamics of strongly coupled chiral gauge theories, none have been discovered as of yet. Therefore, our understanding of chiral gauge theories, as derived from experiment, is limited to the perturbative sector; we must rely on theory to access information about the nonperturbative sector.

Chiral gauge theories have been studied extensively in perturbation theory, particularly as the existence of a regulator that preserves the non-anomalous chiral Ward identities at a given order implies that the theory is perturbatively self-consistent. While such studies do not allow us to determine the complete structure of a theory, they can be used as a guide to determine some of the necessary properties of the nonperturbatively defined theory. First and foremost, the nonperturbative regulator must allow for massless fermions in complex fermion representations in order to preserve gauge invariance. It is also known that only chiral gauge theories with anomaly-free fermion representations are self-consistent, as the existence of gauge anomalies results in the loss of unitarity. This property should persist beyond perturbation theory and therefore the nonperturbative regulator must somehow fail for anomalous representations. Lastly, the nonperturbative regulator should reproduce perturbative results for weakly coupled gauge fields such as the ability to couple only left-handed fermions (in anomaly-free representations) to gauge fields of trivial topology.

Constructing a nonperturbative regulator comes down to the question of how to define the fermion functional integral, $\Delta(A)$

$$
\Delta(A) \equiv \int D \psi D \bar{\psi} e^{-\int d^{2 n} x \bar{\psi} \mathscr{D} \psi} \quad n=1,2, \ldots
$$

in an unambiguous way. This can be done for Dirac fermions where the fermion operator is the Dirac operator $\not D$, which maps from the vector space of left-handed (LH) and right-handed (RH) fermions to the same vector space. Therefore, the theory has a well-posed eigenvalue problem and $\Delta(A)=\operatorname{det}(\not D)$. For chiral gauge theories, it seems natural to take $\mathscr{D}=\not D P_{L}$, where $P_{L}=$ $\left(1-\Gamma_{2 n+1}\right) / 2$ is the projector onto LH fermions. However, this operator does not have a wellposed eigenvalue problem as it maps from the vector space of LH fermions to the vector space of RH fermions. While $|\Delta(A)|$ for such an operator is completely determined, the phase of $\Delta(A)$ cannot be without additional information [1]. The question of how to nonperturbatively define chiral gauge theories is the question of how to define $\mathscr{D}$ such that it acts on Dirac fermions, as is necessary to have an eigenvalue equation, while simultaneously reproducing perturbative results.

Given that the only nonperturbative regulator that has been successfully used for non-integrable QFT is the lattice, it is necessary to ask whether it is possible to define a lattice regularization such that these requirements are satisfied. Two problems immediately present themselves: the no-go theorem of Nielsen and Ninomiya [2] and the fact that symmetries cannot be broken anomalously 


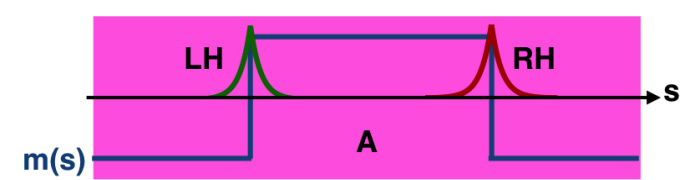

Figure 1: Conventional DWF construction, with light fermions localized at $s=0$ and $s=$ $\pm L$ and a flat profile for the gauge field $A$.

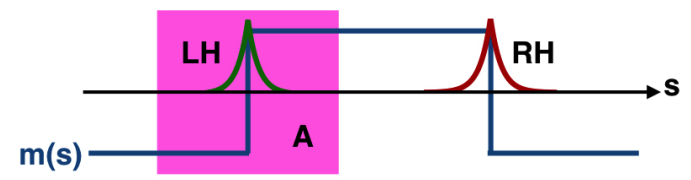

Figure 2: Waveguide model where gauge field is only nonzero near the $s=0$ surface.

on the lattice. While these problems have been overcome for vector theories without the introduction of any new physics below the lattice cutoff scale, it may not be possible to do so for chiral gauge theories. Therefore, finding a nonperturbative regulator for chiral gauge theory may be more than a technical question; instead, the regulator itself may hint at new physics hidden within the Standard Model itself.

\section{Vector Gauge Theories on the Lattice}

The difficulty in implementing massless fermions on the lattice is due to a no-go theorem that forbids the existence of a local fermion operator that describes a single massless Dirac fermion in the continuum while simultaneously preserving all chiral symmetry at finite lattice spacing. Additionally, while anomalous symmetry violation occurs in the continuum, symmetries on the lattice cannot be anomalously broken due to the presence of only a finite number of degrees of freedom. These two problems can be simultaneously overcome in vector theories by realizing that in order to reproduce the physics of the anomalous violation of $U(1)_{A}$ in the continuum, the symmetry has to be broken explicitly in the lattice formulation, in keeping with the no-go theorem. Ginsparg and Wilson argued [3] that for this theory, the fermion operator obeys

$$
\Gamma_{2 n+1} \mathscr{D}+\mathscr{D} \Gamma_{2 n+1}=a \mathscr{D} \Gamma_{2 n+1} \mathscr{D}
$$

where $a$ is the lattice spacing. It was later proven that not only does a solution to the GinspargWilson (GW) equation correctly reproduce the continuum index theorem, it also obeys an exact symmetry that ensures multiplicative mass renormalization even at finite lattice spacing $[4,5]$.

An explicit solution to Eq. 2.1 can be found in the Domain Wall Fermion (DWF) construction [6], where $(2 n+1)$-dimensional fermions have a mass that depends on the extra dimension

$$
m(s)=\left\{\begin{array}{cc}
-m_{-} & s<0 \\
m_{+} & s>0
\end{array}\right.
$$

with $s=[-L, L]$ the coordinate of the compact extra dimension and $m_{ \pm}$is positive. The spectrum of the theory contains light fermions localized at $s=0$ and $s= \pm L$ as well as heavy fermions delocalized into the bulk of the extra dimension. Due to the exponential localization of the light fermions, their mass goes to zero exponentially fast with the size of the extra dimension. In this limit, the mode at $s=0$ is a LH fermion while the mode at $s= \pm L$ is a RH fermion. As any mass renormalization must be proportional to the overlap of these two modes, multiplicative mass 
renormalization is assured. The heavy fermions remain delocalized, and thus massive. Note that flipping the sign of both $m_{+}$and $m_{-}$results in RH fermions localized as $s=0$ and LH at $s=L$.

The theory is gauged by coupling the $(2 n+1)$-dimensional fermions to an $s$-independent $2 n$ dimensional gauge field, as shown in Fig. 1. This ensures a vector-like theory, as both LH and RH fermions couple to the gauge field identically. The explicit violation of $U(1)_{A}$ is due to the bulk fermions, which have large masses. Integrating out these heavy fermions gives rise to a Chern-Simons operator. The incomplete decoupling of the heavy modes, called the Callan-Harvey mechanism [7], allows the $U(1)_{A}$ anomaly to be correctly reproduced at finite lattice spacing.

The fermion functional integral $\Delta(A)$ for this construction can be found in two different ways. The first treats the extra dimension as imaginary time, allowing for a Hamiltonian approach $[8,9]$. Taking $L \rightarrow \infty$ corresponds to projecting onto the groundstates of the Hamiltonians

$$
H_{+}=\Gamma_{2 n+1}\left(D_{W}-m_{+}\right) \quad H_{-}=\Gamma_{2 n+1}\left(D_{W}+m_{-}\right)
$$

where $D_{W}$ is the Wilson operator. Therefore the path integral is given by

$$
\Delta(A)=\frac{\langle\Omega,+\mid \Omega,-\rangle\langle\Omega,-\mid \Omega,+\rangle}{\langle\Omega,+\mid \Omega,+\rangle\langle\Omega,-\mid \Omega,-\rangle}
$$

where $|\Omega, \pm\rangle$ are the multiparticle groundstates of $H_{ \pm}$. The denominator in Eq 2.4 is due to PauliVilars fields which must be introduced to control divergences resulting from the large number of heavy bulk fermions, which become infinite in the large $L$ limit. It is clear that there is no phase ambiguity, as individual phase rotations on the basis vectors of $H_{ \pm}$do not change the form of $\Delta(A)$.

The other method utilizes the slab formulation [10] and treats the discretized extra dimension as a flavor index. Integrating out the bulk flavors allows for the derivation of the effective operator of the boundary fermions explicitly [11]:

$$
\mathscr{D}_{v}^{(L)}=1+\Gamma_{2 n+1} \frac{1-T^{L}}{1+T^{L}}
$$

where $T$ is the transfer matrix responsible for translations in the extra dimension. Taking the large $L$ limit, the effective operator becomes

$$
\mathscr{D}_{V}=1+\Gamma_{2 n+1} \varepsilon\left[H_{+}\right] \quad \varepsilon[H] \equiv \frac{H}{\sqrt{H^{2}}},
$$

where $\varepsilon[H]$ is a matrix sign function. The determinant of $\mathscr{D}_{V}$ and $\Delta(A)$ as derived in Eq. 2.4 agree if $m_{-}$is taken to infinite. This operator satisfies the GW equation and so violates chiral symmetry in a minimal way, just enough to reproduce the effects of the $U(1)_{A}$ anomaly.

\section{Proposal for Lattice Regulated Chiral Gauge Theories}

As the DWF construction gives a method for separating LH from RH fermion, it seems like a good starting point for implementing chiral gauge theories on the lattice. If the $2 n$-dimensional gauge field is given an $s$-dependent profile, opposite chirality partners have different gauge interactions. A previous proposal, shown in Fig. 2, turned off the gauge field half-way between the two 


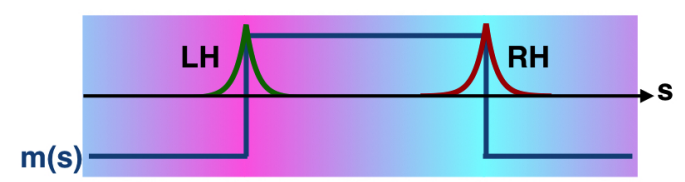

Figure 3: Chiral theory construction where bulk gauge field is solution to gradient flow equation, flowing smoothly from $A$ to $A_{\star}$

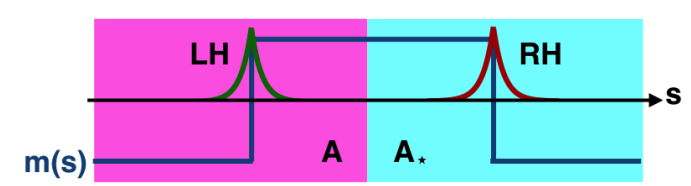

Figure 4: Chiral theory construction where bulk gauge field flows abruptly from $A$ to $A_{\star}$

boundaries, resulting in the gauge-variant theory. Yukawa couplings were introduced at the interface to restore gauge invariance. However, numerical tests indicated that this theory had vector-like interactions, as the Yukawa couplings created a new domain wall [12]. Ref. [13] recently proposed localizing the $2 n$-dimensional gauge field around $s=0$ in a gauge-invariant manner, shown in Fig. 3. Specifically, the gauge field in the extra dimension obeys the gradient flow equation, ${ }^{1}$

$$
\partial_{s} \mathscr{A}_{\mu}(x, s)=\frac{\varepsilon(s)}{\Lambda} D_{v} \mathscr{F}_{v \mu} \quad \mathscr{A}_{\mu}(x, 0)=A(x)_{\mu} \quad \mu, v=1,2, \ldots 2 n
$$

where $\mathscr{F}_{\mu \nu}$ is the field strength tensor defined in terms of $\mathscr{A}_{\mu}(x, s)$ and $A_{\mu}$ is the integration variable in the path integral measures; for simplicity, the domain wall masses $m_{ \pm}$are set equal to $\Lambda$. The gradient flow equation acts like a heat equation, dampening out the high momentum modes of the gauge field. This results in the fermions localized at $s= \pm L$ effectively coupling with exponentially soft form factors. Gauge invariance is maintained as the equation is gauge-covariant.

The properties of the gradient flow equation can be demonstrated in 2-dimensional QED. The gauge field decomposes as

$$
\mathscr{A}_{\mu}(x, s)=\partial_{\mu} \omega(x, s)+\varepsilon_{\mu v} \partial_{v} \lambda(x, s)
$$

where $\omega$, the gauge degree of freedom, shifts under gauge transformations and $\lambda$, the physical degree of freedom, does not; this decomposition also ignores fields of nontrivial topology. Each degree of freedom satisfies its own flow equation,

$$
\partial_{s} \omega(p, s)=0 \quad \partial_{s} \lambda(p, s)=\frac{\varepsilon(s)}{\Lambda} p^{2} \lambda(p, s) .
$$

Notice that fermions at both $s=0$ and $s=L$ couple identically to the gauge degree of freedom while the fermions at $s= \pm L$ couple to the physical degree of freedom with form factor $e^{-p^{2} L / \Lambda}$ which tends to zero as $L \rightarrow \infty$. Therefore, it seems plausible that in the limit of an infinite extra dimension, $\mathrm{RH}$ fermions decouple from the spectrum of the theory in a gauge-invariant manner.

The construction has the necessary road to failure for anomalous fermion representations due to the existence of nonlocality in the low energy $2 n$-dimensional effective theory. Similar to the $U(1)_{A}$ symmetry, the Callan-Harvey mechanism results in a Chern-Simons action

$$
S_{3}=c_{3} \frac{\Lambda}{|\Lambda|} \int d^{2} x d s(\varepsilon(s)-1) \operatorname{Tr}\left[\mathscr{F} \wedge \mathscr{A}-\frac{1}{3} \mathscr{A} \wedge \mathscr{A} \wedge \mathscr{A}\right]
$$

\footnotetext{
${ }^{1}$ For simplicity, we work with the continuum gradient flow equation for now.
} 
where $\varepsilon(s)$ is due to the fermions, $(-1)$ is due to the Pauli-Villars and $c_{3}$ is a known coefficient. Focusing on 2-dimensional QED, Eq 3.4 can be evaluated by noticing that the Tr term is a total $s$-derivative and so, upon integration by parts, only the surface terms at $s=0, \pm L$ contribute. This results in a nonlocal 2-dimensional action,

$$
S_{C S}=2 c_{3} \sum_{i} q_{i}^{2} \frac{\Lambda_{i}}{\left|\Lambda_{i}\right|} \int d^{2} x d^{2} y\left(\frac{\partial_{\mu} \partial_{\alpha}}{\partial^{2}} A_{\mu}(x)\right) \Gamma(x-y)\left(\frac{\partial_{\mu} \partial_{\beta}}{\partial^{2}} \varepsilon_{\beta \gamma} A_{\gamma}(x)\right)
$$

where the sum is over fermion species with gauge coupling $q_{i}$ and domain wall mass $\Lambda_{i}$. As long as this operator is present, the 2-dimensional theory is nonlocal. However, this term vanishes if

$$
\sum_{i} q_{i}^{2} \frac{\Lambda_{i}}{\left|\Lambda_{i}\right|}=0
$$

and locality is restored. Since $\Lambda /|\Lambda|$ is the chirality of the fermion sitting at $s=0$, the sum only vanishes for fermion representations that are gauge-anomaly free in the continuum; an analogous criteria holds in in higher dimensions, as the Chern-Simons prefactor depends on dimension. Therefore to achieve a local $2 n$-dimensional theory, the fermions must be in an anomaly-free representation and the road to failure for this construction of chiral gauge theories is loss of locality.

The proposal of Ref. [13] was formulated at finite $L$. However, the spectrum of DWF at finite lattice spacing only has exactly massless modes in the limit of an infinite extra dimension. In such a limit the methods developed for the vector case can be used to derive the effective operator for this construction. Ref. [14] does this specifically in the simplifying scenario where all the change in the gauge field occurs rapidly as shown in Fig. 4. In this 'abrupt flow' scenario, there are only two gauge fields of importance,

$$
A_{\mu}(x)=\mathscr{A}_{\mu}(x, 0) \quad A_{\star}^{\mu}(x) \equiv \lim _{L \rightarrow \infty} \mathscr{A}^{\mu}(x, L) .
$$

where $A_{\star}$ is the attractive fixed point solution to the gradient flow equation. Defining the sign functions for the Hamiltonians evaluated with these two gauge fields,

$$
\varepsilon \equiv \varepsilon\left[\Gamma_{2 n+1}\left(D_{W}(A)-m_{+}\right)\right] \quad \varepsilon_{\star} \equiv \varepsilon\left[\Gamma_{2 n+1}\left(D_{W}\left(A_{\star}\right)-m_{+}\right)\right]
$$

the effective operator for a $\mathrm{LH}$ fermion coupling to the gauge field $A$ and a $\mathrm{RH}$ fermion coupling to the gauge field $A_{\star}$ is

$$
\mathscr{D}_{\chi}=1+\Gamma_{2 n+1}\left[1-\left(1-\varepsilon_{\star}\right) \frac{1}{1+\varepsilon \varepsilon_{\star}}(1-\varepsilon)\right]
$$

for the case where $\operatorname{Tr} \varepsilon=\operatorname{Tr} \varepsilon_{\star}$.

The operator in Eq. 3.9 satisfies several important properties. It is a solution to the GinspargWilson equation, indicating that the fermion mass is multiplicatively renormalized and the $U(1)_{A}$ symmetry is explicitly violated, as needed to give the correct $U(1)_{A}$ anomaly. The determinant of this operator also does not have a phase ambiguity, as it can be treated in the same Hamiltonian approach as the vector case. Lastly, the operator has the continuum limit

$$
\lim _{a \rightarrow 0} \mathscr{D}_{\chi}=\left(\begin{array}{cc}
0 & D(A)_{\mu} \sigma^{\mu} \\
D\left(A_{\star}\right) \bar{\sigma}^{\mu}
\end{array}\right)
$$


as would be expected from the setup shown in Fig. 4. If $A_{\star}$ is pure gauge then the RH fermions decouple completely from physical degrees of freedom. However, the continuum gradient flow equation has multiple attractive fixed points that correspond to gauge fields of different winding numbers. For these gauge fields, $A_{\star}$ is not pure gauge but in fact contains topological gauge configurations such as instantons. As these are physically relevant degrees of freedom, it would seem that the RH fermions do not decouple from the physical spectrum of the theory. It is not fully understood whether these couplings persist for discretized flow equations nor whether their contribution to the action is extensive. However, the existence of such couplings is a radical departure from how nonperturbatively regulated chiral gauge theories are typically expected to behave and so it seems imperative to ask what the phenomenological implications of such topological couplings could be.

\section{References}

[1] Luis Alvarez-Gaume and Paul H. Ginsparg, The Topological Meaning of Nonabelian Anomalies, Nucl. Phys. B243 (1984) 449

[2] Holger B. Nielson and Masao Ninomiya, No Go Theorem for Regularizing Chiral Fermions, Phys. Lett. B105 (1981) 219

[3] Paul H. Ginsparg and Kenneth G. Wilson, A remnant of chiral symmetry on the lattice, Phys. Rev. D25 (1982) 2649

[4] Peter Hasenfratz, Victor Laliena, and Ferenc Niedermayer, The Index theorem in QCD with a finite cutoff, Phys. Lett. B427 (1998) 125 [hep-lat/9801021]

[5] Martin Lüscher, Exact chiral symmetry on the lattice and the Ginsparg-Wilson relation, Phys. Lett. B428 (1998) 342 [hep-lat/9802011]

[6] David B. Kaplan, A method for simulating chiral fermions on the lattice, Phys. Lett. B288 (1992) 342 [hep-lat/9206013]

[7] Curtis G. Callan, Jr. and Jeffrey A. Harvey, Anomalies and fermion zero modes on strings and domain walls, Nucl. Phys. B250 (year) page

[8] Rajamani Narayanan and Herbert Neuberger, Chiral determinant as an overlap of two vacua, Nucl. Phys. B412 (1994) 574 [hep-lat/93070 06]

[9] Rajamani Narayanan and Herbert Neuberger, A Construction of lattice chiral gauge theories, Nucl. Phys. B443 (1995) 305 [hep-th/9411108]

[10] Yigal Shamir, Chiral fermions from lattice boundaries, Nucl. Phys. B406 (1993) 90 [hep-lat/9303005]

[11] Herbert Neuberger, Vector-like gauge theories with almost massless fermions on the lattice, Phys. Rev. D57 (1998) 5417 [hep-lat/9710089]

[12] Maarten Golterman, Karl Jansen, Donald N. Petcher and Jeroen C. Vink, Investigation of the domain wall fermion approach to chiral gauge theories on the lattice, Phys. Rev. D49 (1994) 1607 [hep-lat/9309015]

[13] Dorota M. Grabowska and David B. Kaplan, Nonperturbative regulator for chiral gauge theories?, Phys. Rev. Lett. 116 (2016) 211602 [1511.03649]

[14] Dorota M. Grabowska and David B. Kaplan, A Chiral Solution to the Ginsparg-Wilson Equation, [1610.02151] 